\title{
剪切稀化对径向滑动轴承润滑特性的影响*
}

\author{
裴世源 郑文斌 洪 军 \\ (西安交通大学现代设计与转子轴承系统教育部重点实验室 西安 710049)
}

\begin{abstract}
摘要: 为研究润滑油的剪切稀化效应对径向滑动轴承性能的影响, 考虑剪切稀化、紊流、质量守恒边界建立了轴承的热流体 动力学润滑分析模型, 采用有限元法求解了其润滑性能, 通过对比理论与试验结果验证了理论分析模型的正确性, 分析了不 同转速、载荷和进油温度下剪切稀化对轴承润滑性能的影响规律(油膜厚度、油膜压力、温升、功耗、流量等), 探讨了温度 计算模型对剪切稀化效应的适用性。结果发现, 剪切稀化效应对轴承润滑特性具有重要影响, 其作用强弱与载荷和转速等条件 强烈相关; 轻载工况剪切稀化对轴承润滑特性体现为正面作用, 重载工况体现为负面作用; 发现温度计算模型对选用不当和定 偏心计算是造成功耗等理论计算结果与试验之间存在较大偏差的两个主要原因。最后给出了工程上选用具有剪切稀化效应润滑 油的适用工况与条件。
\end{abstract}

关键词: 剪切稀化; 非牛顿流体; 润滑特性; 热流体动力学

中图分类号: TP301

\section{Influence of Shear-thinning Effect on the Characteristics of Journal Film Bearing}

\author{
PEI Shiyuan ZHENG Wenbin HONG Jun \\ (Key Laboratory of Education Ministry for Modern Design and Rotor-bearing System, \\ Xi'an Jiaotong University, Xi'an 710049)
}

\begin{abstract}
The purpose of this paper is to study the lubricant shear-thinning effects on the film bearing performance. A thermal-hydrodynamic lubrication analysis model is presented, considering the shear-thinning effect, turbulent effect, and mass conserving cavitation boundary conditions. Then, the bearing performance is solved by finite element method. The correctness of the analytical model is verified by the comparison between the theoretical and existing experimental data. Under wide operating conditions, such as speeds, loads and oil supply temperatures, the effects of shear thinning on the lubrication performance, such as film thickness, film pressure, temperature rise, power consumption, flow rate, is systematically investigated. The applicability of the thermal model is discussed. It is showed that the shear thinning effect has important influence on the film bearing performance. The significance of the shear thinning effect is depending on the load and speed conditions. Shear thinning effects are improved the bearing performance in light load condition and deteriorate in heavy load condition. The thermal model is critical to correctly evaluate of shear thinning effects on the bearing performance, which is the reason that large deviation between theory and experiment was observed. Finally, the preferred working conditions for the lubricant with shear thinning effect is suggested.
\end{abstract}

Key words: shear thinning effect; non-Newtonian fluid; lubrication characteristic; thermohydrodynamics

\section{0 前言}

为改善油品性能, 目前工业润滑油如车用机油、 齿轮油、液压油以及主轴油等均含有不同比例的各 种高分子添加剂, 某些润滑油中添加剂含量最高可 达 $20 \%{ }^{[1]}$ 。高分子添加剂可显著改变润滑油的流变

* 国家自然科学基金资助项目 (51605367, 51975456)。20190205 收到初稿, 20190815 收到修改稿
特性, 使其变为非牛顿流体, 主要表现为剪切稀化 效应, 即润滑油的黏度随剪切率的升高而降低。由 于低速下润滑油黏度高, 易形成油膜, 而高速下黏 度下降, 可降低功耗和温升, 故一般认为剪切稀化 效应对轴承性能具有正面作用。国内外学者对润滑 油的剪切稀化效应及其对轴承性能的影响进行了长 期而广泛的研究。根据所采用的模型与方法的不同, 大致可将研究分为三个阶段：早期研究主要采用幂 律模型描述剪切稀化效应, 并研究其对轴承性能的 影响; 随后, 为克服幂律模型适用范围窄等问题, 
学者提出了新的本构方程如 GECIM 模型 ${ }^{[2]}$ 并研究 其对轴承性能的影响; 近期, 人们开始综合考虑更 多因素(如润滑油的压黏特性、黏弹性、混合润滑等) 及耦合作用下剪切稀化效应对轴承的影响。

早期研究中所采用的幂律模型的方程简单、计 算方便。如 TANNER ${ }^{[3]}$ 首次采用幂律模型和短轴承 理论提出了考虑非牛顿效应的油膜压力解析解。 WADA 等 ${ }^{[4-5]}$ 对具有不同含量高分子添加剂润滑 油的径向轴承的压力分布进行了理论和试验研究, 结果表明添加剂含量越高剪切稀化效应越强。

SWAMY 等采用等温模型先后研究了非牛顿效应 对径向轴承静特性 ${ }^{[6]}$ 、动特性 ${ }^{[7]}$ 和稳定性 ${ }^{[8]}$ 的影响, 结果显示剪切稀化使轴承的承载力、刚度、阻尼和 稳定性均有所下降。DIEN 等 ${ }^{[9]}$ 推导了基于幂律模 型的广义雷诺方程。JIIN-YUH 等 ${ }^{[10-11]}$ 采用幂律本 构和绝热模型各自研究了定偏心下非牛顿流体润 滑下径向轴承的润滑特性, 结果显示剪切稀化使温 度、功耗和承载力都有所下降。ZHANG 等 ${ }^{[1]}$ 基于 ISOADI 热分析模型和质量守恒算法研究了定偏心 下剪切稀化效应对具有粗糙表面的动载轴承润滑 性能的影响。

随后人们发现幂律本构方程的适用范围较为有 限, 例如当剪切速率很小时计算黏度趋近于无穷大, 而当剪切速率很大时黏度趋近于零。而实际上在这 两种情况下, 润滑油黏度趋近于恒定的有限值。为 克服幂律模型的缺点, $\mathrm{GECIM}^{[2]}$ 提出了一种更准确 的描述剪切稀化效应本构方程, 其本质上是 $\mathrm{CROSS}^{[12]}$ 模型的一种变形, 作者指出剪切稀化效应 可以降低约 $40 \%$ 的承载力和摩擦功耗。PARANJPE 等 ${ }^{[13]}$ 使用 GECIM 模型结合质量守恒算法研究了剪 切稀化对动载荷轴承润滑性能的影响, 结果显示剪 切稀化造成动载轴承的摩擦功耗显著减小, 最小油 膜厚度明显降低。WANG 等 ${ }^{[14]}$ 采用平均剪应变率和 GECIM 模型并结合质量守恒算法研究了剪切稀化 特性对动载荷轴承的润滑性能的影响, 结果显示剪 切稀化使最大油膜压力增加 $25 \%$, 功耗降低 $24 \%$ 。 JANG 等 ${ }^{[15]}$ 基于 GECIM 模型和两维的 THD 方法系 统地研究了零剪切黏度、无穷剪切黏度和稳定性参 数对压力分布和温度分布的影响。FATU 等 ${ }^{[16]}$ 采用 GECIM 模型和广义雷诺方程分析了剪切稀化对发 动机连杆轴承性能的影响, 发现油膜厚度显著降低。 BUKOVNIK 等 ${ }^{[17]}$ 使用 AVL 软件基于 CROSS 本构 方程研究了剪切稀化对动载轴承的影响, 结果表明 在高速下剪切稀化使黏度可降低 50\%, 摩擦功耗可 降低 30\%。

近年来, 环保要求日益严格, 为减小排放并降
低功耗, 各种含有高分子添加剂的润滑油应用更加 广泛, 人们对剪切稀化效应的研究也愈加重视。2014 年, 张振山 ${ }^{[18]}$ 研究了剪切稀化对动载轴承润滑性能 的影响, 发现剪切稀化使轴承的最高温度下降。2015 年, SANDER 等 ${ }^{[19]}$ 研究了剪切稀化与压黏效应及其 耦合因素对轴承摩擦的影响, 结果显示对于含高分 子添加剂的润滑油忽略剪切稀化效应会显著高估摩 擦功耗。2016 年, 宁李谱等 ${ }^{[20]}$ 研究了剪切稀化效应 对发动机活塞裙部润滑性能的影响, 结果显示剪切 稀化效应可以降低功耗, 但也减小了最小油膜厚度, 增加了产生磨损的风险。2017 年, 汤占岐 ${ }^{[21]}$ 研究了 剪切稀化对关节轴承的影响, 发现流变指数较高的 润滑剂具有较好的润滑性能。2018 年, MARX等 ${ }^{[22-24] ~}$ 连续发表了多篇关于润滑油剪切稀化效应的论文, 他测量了 ${ }^{[22]}$ 高分子添加剂对流变特性影响并提出 了新的流变本构方程, 理论研究 ${ }^{[23]}$ 表明剪切稀化效 应最多可以降低 $50 \%$ 的摩擦功耗, 其试验结果 ${ }^{[24]}$ 也证实剪切稀化效应具有一定的降低功耗的作用, 但降耗幅度远未达到理论分析的程度。

综上, 虽然人们对润滑油的剪切稀化效应进行 了较充分地研究, 但目前在理论和应用方面依然存 在两方面的问题: 其一, 工程上对何时选用具有剪 切稀化效应的润滑油还未达成共识。已有研究结果 表明剪切稀化对轴承可以降低功耗和温升, 但也可 减小最小油膜厚度和承载力, 故润滑油的剪切稀化 对轴承性能同时存在正面与负面的影响, 这给工程 人员何时选用具有剪切稀化效应的润滑油带来了 困扰。其二, 对于剪切稀化对轴承摩擦功耗的影响, 理论与试验结果存在较大偏差。大量理论计算说明 剪切稀化效应可以显著降低摩擦功耗, 最多可达 $30 \% \sim 50 \%$ 以上 ${ }^{[2,16-17,23]}$, 这显然对工业界具有重 大价值, 例如水轮机主推力轴承的摩擦功耗降低 $3 \% \sim 5 \%$ 意味着每天可降低数百万千瓦的能量损 耗, 避免数十万元的经济损失, 然而目前已有的试 验数据 ${ }^{[5,19-20,22,24]}$ 并没有证明剪切稀化确具有如 此显著地降低功耗的作用。

针对上述两个问题, 本文综合考虑剪切稀化效 应、紊流、质量守恒边界等因素建立热流体动力学 润滑分析模型, 基于初始黏度相同的有无剪切稀化 效应的润滑油, 研究不同工况下径向轴承关键润滑 性能(油膜厚度、油膜压力、温升、功耗、流量等) 的变化, 通过理论与试验对比验证理论分析模型的 正确性，揭示不同工况条件下剪切稀化效应的对轴 承性能的影响规律, 探讨目前理论计算结果与试验 结果存在偏差的原因, 给出对具有剪切稀化效应润 滑油的适用工况和选用准则。 


\section{1 理论基础}

\section{1 控制方程}

考虑瞬态、剪切稀化效应、紊流、质量守恒的 流体在微小间隙的运动可用如下雷诺方程(1)进行 描述 ${ }^{[14,25]}$

$$
\begin{gathered}
\frac{\partial}{\partial x}\left(\frac{G_{x} \rho h^{3}}{\eta} \frac{\partial p}{\partial x}\right)+\frac{\partial}{\partial y}\left(\frac{G_{y} \rho h^{3}}{\eta} \frac{\partial p}{\partial y}\right)=\frac{U}{2} \frac{\partial(\rho h)}{\partial x}+\frac{\mathrm{d}(\rho h)}{\mathrm{d} t} \\
G_{x}=\frac{1}{12+0.0136 R e^{0.90}} \\
G_{y}=\frac{1}{12+0.0043 R e^{0.96}} \\
h=c-e_{x} \cos \theta-e_{y} \sin \theta
\end{gathered}
$$

式中, $x=R \theta, R$ 为轴承半径, $\theta$ 为周向角度; $y$ 为轴 向坐标; $\eta$ 为润滑油黏度; $\rho$ 为润滑油密度; $p$ 为油 膜压力; $G_{x}$ 和 $G_{y}$ 分别是周向和轴向的紊流修正因 子, 由式(2)和(3)计算; $R e$ 为当地雷诺数, 由式(4) 计算; $h$ 为油膜厚度, 由式(5)计算; $c$ 是轴承半径 间隙; $e_{x}$ 和 $e_{y}$ 分别是轴颈水平和坚直方向的位移。

热效应是油膜轴承润滑分析必须考虑的因素. 目前计算油膜温度场主要具有三种模型：其一是根 据功耗和流量计算等效工作温度的热平衡方法, 该 方法计算速度快, 但不能得到最高温度等关键性能 参数, 固定瓦轴承单工况(通常需要迭代求解雷诺方 程数百次)计算时间通常小于 $5 \mathrm{~s}$ 。其二是忽略油膜 方向温度梯度的绝热模型, 该能量方程是二维空间, 可与雷诺方程共用一套网格, 需要迭代求解油膜温 度和油池温度, 单工况计算时间通常小于 $15 \mathrm{~s}$; 其 三是考虑固体导热和油膜厚度方向温度变化的三维 导热模型, 该模型需要同时建立轴颈、油膜和轴瓦 的三维网格模型, 单工况计算时间通常需要数十分 钟, 然而, 由于需要更多的输入参数且通常难以获 得准确的边界条件, 如瓦背环境温度和对流换热系 数等, 三维导热模型并不能保证计算结果更精确, 在实际工业轴承设计中也较少采用。

为平衡计算时间与精度, 本文采用绝热模型考 虑轴承的热效应, 能量方程 ${ }^{[25]}$ 如式(6)所示

$$
\begin{gathered}
\rho c_{p}\left[q_{x} \frac{\partial T}{\partial x}+q_{y} \frac{\partial T}{\partial y}\right]= \\
\frac{\partial}{\partial x}\left(k \frac{\partial T}{\partial x}\right)+\frac{\partial}{\partial y}\left(k \frac{\partial T}{\partial y}\right)-q_{x} \frac{\partial p}{\partial x}-q_{y} \frac{\partial p}{\partial y}+\tau U
\end{gathered}
$$

$$
\begin{gathered}
q_{x}=\frac{U h}{2}-\frac{G_{x} h^{3}}{\eta} \frac{\partial p}{\partial x} \\
q_{y}=-\frac{G_{y} h^{3}}{\eta} \frac{\partial p}{\partial y} \\
\tau=C_{f} \frac{\eta U}{h}+\frac{h}{2} \frac{\partial p}{\partial x} \\
C_{f}=1+0.0012 R e^{0.94}
\end{gathered}
$$

式中, $T$ 为沿油膜厚度方向的平均温度; $c_{p}$ 为润滑 油的比热容; $k$ 为润滑油的热导率; $q_{x}$ 和 $q_{y}$ 分别为 计算域各点周向和轴向的体积流量, 分别由式(7)和 式(8)计算; $\tau$ 为作用在轴颈上的切向应力, 由式(9) 计算; $C_{f}$ 为由于紊流所造成的切向应力修正因子, 由式(10)计算; $U$ 为轴颈线速度。

油膜在水平和重力方向的承载力由式(11)确 定, 瓦块两侧的流量由式(12)计算, 上下游的流量 由式(12)和式(13)计算, 摩擦力由式(15)计算, 功耗 由式(16)计算。

$$
\begin{gathered}
\left\{\begin{array}{c}
F_{h} \\
F_{v}
\end{array}\right\}=\int_{-B / 2}^{B / 2} \int_{0}^{L} p\left\{\begin{array}{l}
\cos \theta \\
\sin \theta
\end{array}\right\} \mathrm{d} x \mathrm{~d} y \\
\left.Q\right|_{y= \pm B / 2}=\left.\int_{0}^{L} q_{y}\right|_{y= \pm B / 2} \mathrm{~d} x \\
\left.Q\right|_{x=0}=\left.\int_{-B / 2}^{B / 2} q_{x}\right|_{x=0} \mathrm{~d} x \\
\left.Q\right|_{x=L}=\left.\int_{-B / 2}^{B / 2} q_{x}\right|_{x=L} \mathrm{~d} y \\
F_{t}=\int_{-B / 2}^{B / 2} \int_{0}^{L} \tau \mathrm{d} x \mathrm{~d} y \\
P=F_{t} U
\end{gathered}
$$

\section{2 边界条件}

雷诺方程瓦块上下游的压力设定为供油压力, 瓦块两侧的压力为零, 分别由式(17)和式(18)确定。

$$
\begin{gathered}
\left.p\right|_{x=0}=\left.p\right|_{x=L}=p_{s} \\
\left.p\right|_{y= \pm B / 2}=0
\end{gathered}
$$

对于油膜空化现象, 本文采用 KUMAR 等 ${ }^{[26]}$ 所提 出的 JFO 质量守恒边界计算油膜破裂和再形成边界, 由式(19)和式(20)确定，其中 $n$ 表示界面法向方向。

$$
\begin{gathered}
p=0 \quad \frac{\mathrm{d} p}{\mathrm{~d} x}=0 \\
\frac{h^{2}}{12 \eta} \frac{\partial p}{\partial n}=\frac{U_{n}}{2}\left(1-\theta_{n}\right)
\end{gathered}
$$

需要注意的是采用 JFO 质量守恒边界需要同 时求解压力场、密度场和黏度场, 密度场和黏度场 满足式(21)和式(22)的关系, $r$ 定义为油膜占空比, 其中 $\rho_{\mathrm{liq}}$ 和 $\eta_{\mathrm{liq}}$ 分别表示润滑油没有空化时的密度 与黏度。 


$$
\begin{gathered}
0 \leqslant \rho \leqslant \rho_{\text {liq }} \\
0 \leqslant \eta \leqslant \eta_{\text {liq }} \\
r=\frac{\eta}{\eta_{\text {liq }}}=\frac{\rho}{\rho_{\text {liq }}}
\end{gathered}
$$

同时, 虽然能量方程包含二阶热传导项, 但在 绝大多数情况下式(6)左端对流项占主导地位, 因此 瓦块的入口温度边界条件至关重要。从上瓦下游流 出的热油会进入到油槽中与新油混合进入下瓦的入 口, 故瓦块入口和出口边的温度往往显著高于进油 温度, 本文采用热量守恒方法计算瓦块入口边的温 度, 由式(23)确定, 其中 $\lambda$ 是热回油因子, 本文设 置为 1 , 表示从上瓦流出的热油全部进入到下瓦。

$$
T_{\text {inlet }}=\frac{\lambda Q_{\text {out }} T_{\text {out }}+\left(Q_{\text {in }}-\lambda Q_{\text {out }}\right) T_{\text {supply }}}{Q_{\text {in }}}
$$

瓦块下游和两侧的边界条件施加绝热条件, 即 由式(24)确定。

$$
\left.\frac{\partial T}{\partial x}\right|_{x=L}=\left.\frac{\partial T}{\partial y}\right|_{y= \pm B / 2}=0
$$

\section{2 润滑油流变模型}

本文选取 WALTHER 模型 ${ }^{[25]}$ 和 CROSS 模型 ${ }^{[12]}$ 相 结合描述润滑油的流变特性。WALTHER 模型 ${ }^{[25]}$ 描述 润滑油的温度和黏度的关系, 由式(25)和(26)确定, 即

$$
\begin{gathered}
\eta_{0}(T)=\rho v_{0} \\
\lg \lg \left(v_{0}+0.7\right)=A-B \lg T
\end{gathered}
$$

式中, $\eta_{0}$ 和 $v_{0}$ 分别为温度 $T$ 下的动力黏度和运动黏 度; 温度单位为 ${ }^{\circ} \mathrm{C}, \rho$ 为润滑油密度; 系数 $A$ 和 $B$ 可根据两点的温度和动力黏度进行求解。

本文采用 CROSS 模型 ${ }^{[12]}$ 描述润滑油的剪切率 与黏度的关系, 即剪切稀化效应由式(27) (30)确定

$$
\begin{gathered}
\eta=\eta_{0}\left[\frac{1+m_{1}\left(\gamma / \gamma_{c}\right)^{m_{0}}}{1+\left(\gamma / \gamma_{c}\right)^{m_{0}}}\right] \\
\gamma=\sqrt{\left\langle\left(\frac{\partial u}{\partial z}\right)^{2}+\left(\frac{\partial v}{\partial z}\right)^{2}\right\rangle} \\
\gamma_{c}=10^{\alpha+\beta T} \\
m_{1}=\eta_{\gamma_{\infty}} / \eta_{\gamma_{0}} \\
u=\left(\frac{z^{2}-z h}{2 \eta}\right) \frac{\partial p}{\partial x}+\frac{z}{h} U \\
v=\left(\frac{z^{2}-z h}{2 \eta}\right) \frac{\partial p}{\partial y}
\end{gathered}
$$

\begin{tabular}{|c|c|}
\hline 项目 & 数值 \\
\hline 密度 $/\left(\mathrm{kg} \cdot \mathrm{m}^{-3}\right)$ & 870 \\
\hline 比热容 $/\left(\mathrm{J} \cdot \mathrm{kg}^{-1} \cdot \mathrm{K}^{-1}\right)$ & 1806 \\
\hline $40{ }^{\circ} \mathrm{C}$ 运动黍度 $/\left(\mathrm{mm}^{2} \cdot \mathrm{s}^{-1}\right)$ & 32.9 \\
\hline $100{ }^{\circ} \mathrm{C}$ 运动黏度 $/\left(\mathrm{mm}^{2} \cdot \mathrm{s}^{-1}\right)$ & 5.6 \\
\hline 剪切稀化因子 $m_{1}$ & $0.50,0.75,1.00$ \\
\hline 剪切稀化系数 $m_{0}$ & $2 / 3$ \\
\hline 式(29)系数 $\alpha$ & 2.5 \\
\hline 式(29)系数 $\beta$ & 0.03 \\
\hline
\end{tabular}

式中, $\gamma$ 为沿油膜厚度平均的剪切率; $u$ 和 $v$ 分别 为润滑油沿周向和轴向的速度; 剪切稀化因子 $m_{1}$ 定义为剪切率无穷大时的动力黏度 $\eta_{\gamma_{x}}$ 与剪切率为 零时动力黏度 $\eta_{\gamma_{0}}$ 的比值, 通常取值范围为 $0.1 \sim 1.0$; $\gamma_{c}$ 为临界剪切率。表 1 列出了以 VG32 为基础油的 某多级润滑油的参数。假定轴颈直径为 $100 \mathrm{~mm}$, 间 隙比为 $0.2 \%$, 无偏心, 图 1 展示了不同温度 $T$ 和剪 切稀化因子 $m_{1}$ 下, 黏度随转速的变化。

表 1 某典型润滑油参数

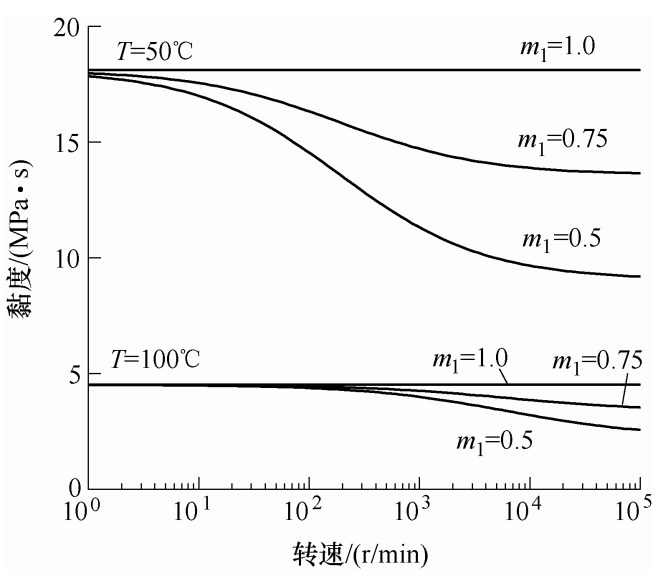

图 1 动力黏度随转速的变化

由式(25) (30)和图 1 可见, 影响黏度的三个主 要因素是温度、转速和剪切稀化因子 $m_{1}$ 。首先, 随 着转速的升高黏度呈现非线性降低, 而变化规律与 温度 $T$ 和剪切稀化因子 $m_{1}$ 均有密切联系。其次, 剪 切稀化因子 $m_{1}$ 是表征润滑油剪切稀化效应的重要参 数之一, 主要影响超过临界剪切率 $\gamma_{\mathrm{c}}$ 后的黏度, 当 $m_{1}$ 为 1.0 时, 表示润滑油没有剪切稀化特性, 而 $m_{1}$ 越小表示剪切稀化效应越强。最后, 温度是影响润 滑油黏度的最重要因素，体现在两方面：其一，温 度升高, 润滑油的基础黏度急剧下降, 例如 $100{ }^{\circ} \mathrm{C}$ 时的基础动力黏度仅是 $50{ }^{\circ} \mathrm{C}$ 的 $1 / 4$; 其二, 温度升 高, 剪切稀化效应的临界剪切率 $\gamma_{\mathrm{c}}$ 显著提高, 例如 $50{ }^{\circ} \mathrm{C}$ 时的转捩速度约为 $200 \mathrm{r} / \mathrm{min}$, 而 $100{ }^{\circ} \mathrm{C}$ 时提高 至约 $6000 \mathrm{r} / \mathrm{min}$, 而由图 1 可见, 在转捩速度附近黏 度快速下降。综上: 剪切稀化效应是剪切率和温度 的强函数, 而剪切率和温度又与工况强烈相关, 故 
剪切稀化效应的强弱会随工况的不同而不同。

\section{3 结果与讨论}

本文采用有限元方法对雷诺方程和能量方程进 行离散, 在周向和轴向采用均布 $320 \times 40$ 四节点等 参元对计算域进行离散, 由于对称性在轴向取一半 求解。使用高斯数值积分计算油膜合力, 摩擦力和 流量。采用 Newton-Raphson 算法迭代求解轴颈水平 和坚直方向上的偏心距、油膜温度、油池温度和油 膜占空比, 迭代收玫精度为 $10^{-3}$, 最终压力场、温 度场、油膜占空比收玫后, 采用小扰动法求动特性 系数。为研究剪切稀化效应对径向滑动轴承性能的 影响, 本文采用第 $1 、 2$ 节的理论模型和润滑油流变 模型, 计算不同转速、载荷和进油温度下剪切稀化 效应对轴承润滑特性的影响规律。同时, 为验证理 论分析模型的正确性, 我们将理论计算结果与试验 测量数据 ${ }^{[27]}$ 进行了对比。

\section{1 算例描述}

某径向滑动轴承的几何参数与工况条件 ${ }^{[27]}$ 如 表 2 所示, 试验所采用的润滑油牌号为 VG32, 不 包含剪切稀化效应, 作对比参照, 具有剪切稀化效 应的润滑油设置剪切稀化因子 $m_{1}=0.5$, 润滑油具 体参数参见表 $1^{[27]}$ 。

\section{表 2 轴承几何与工况参数}

\begin{tabular}{lc}
\hline \multicolumn{1}{c}{ 项目 } & 数值 \\
\hline 直径 $/ \mathrm{mm}$ & 76.2 \\
宽度 $/ \mathrm{mm}$ & 38.1 \\
间隙 $/ \mathrm{mm}$ & 0.0762 \\
瓦块包角 $/\left(^{\circ}\right)$ & 151 \\
瓦块数量 & 2 \\
转速 $/(\mathrm{r} / \mathrm{min})$ & $200 \sim 10000$ \\
载荷 $/ \mathrm{kN}$ & $1 \sim 10$ \\
载荷方向 $/\left(^{\circ}\right)$ & 270 \\
润滑油牌号 & $\mathrm{VG} 32$ \\
进油温度 $/{ }^{\circ} \mathrm{C}$ & $20 \sim 70$ \\
供油压力 $/ \mathrm{MPa}$ & 0.2 \\
\hline
\end{tabular}

\section{2 典型工况下的静动特性对比}

为验证理论分析模型的正确性并初步研究剪切 稀化对轴承性能的影响, 本节对比工作转速为 8000 $\mathrm{r} / \mathrm{min}$, 载荷为 $9430 \mathrm{~N}$, 进油温度为 $50{ }^{\circ} \mathrm{C}$ 时轴承的 静动特性。表 3 对比了有无剪切稀化效应时轴承的 关键润滑特性参数。图 2 展示了无剪切稀化效应的 轴承油膜的压力、温度、厚度和占空比的三维分布。 图 3 对比了中心线处理论与试验的油膜温度。图 4 对比了中心线处有无剪切稀化效应的油膜厚度和油 膜压力。
表 3 关键润滑性能对比

\begin{tabular}{|c|c|c|c|}
\hline 参数 & $\begin{array}{c}\text { 无剪切稀化 } \\
\text { (试验) }\end{array}$ & $\begin{array}{c}\text { 无剪切稀化 } \\
\text { (理论) }\end{array}$ & $\begin{array}{c}\text { 有剪切稀化 } \\
\text { (理论) }\end{array}$ \\
\hline 偏心率 & 0.8 & 0.793 & 0.816 \\
\hline 最小油膜厚度/ $\mu \mathrm{m}$ & 15.2 & 15.5 & 14.0 \\
\hline 最大油膜压力 $/ \mathrm{MPa}$ & - & 11.0 & 12.4 \\
\hline 摩擦功耗/kW & 1.75 & 1.57 & 1.53 \\
\hline 侧泄流量/ $\left(\mathrm{L} \cdot \mathrm{min}^{-1}\right)$ & 2.31 & 3.08 & 3.38 \\
\hline 最高温度/C & 96 & 94.8 & 99.0 \\
\hline $\begin{array}{l}\text { 刚度系数 } \\
k_{x x} /\left(\mathrm{N} \cdot \mathrm{m}^{-1}\right)\end{array}$ & - & $2.00 \times 10^{8}$ & $2.01 \times 10^{8}$ \\
\hline $\begin{array}{l}\text { 刚度系数 } \\
k_{x y} /\left(\mathrm{N} \cdot \mathrm{m}^{-1}\right)\end{array}$ & - & $-6.08 \times 10^{7}$ & $-6.88 \times 10^{7}$ \\
\hline $\begin{array}{l}\text { 刚度系数 } \\
k_{y x} /\left(\mathrm{N} \cdot \mathrm{m}^{-1}\right)\end{array}$ & - & $-4.90 \times 10^{8}$ & $-5.61 \times 10^{8}$ \\
\hline $\begin{array}{l}\text { 刚度系数 } \\
k_{y y} /\left(\mathrm{N} \cdot \mathrm{m}^{-1}\right)\end{array}$ & - & $8.10 \times 10^{8}$ & $9.57 \times 10^{8}$ \\
\hline $\begin{array}{l}\text { 阻尼系数 } \\
c_{x x} /\left(\mathrm{N} \cdot \mathrm{s} \cdot \mathrm{m}^{-1}\right)\end{array}$ & - & $1.63 \times 10^{5}$ & $1.53 \times 10^{5}$ \\
\hline $\begin{array}{l}\text { 阻尼系数 } \\
c_{x y} /\left(\mathrm{N} \cdot \mathrm{s} \cdot \mathrm{m}^{-1}\right)\end{array}$ & - & $-2.36 \times 10^{5}$ & $-2.76 \times 10^{5}$ \\
\hline $\begin{array}{l}\text { 阻尼系数 } \\
c_{y x} /\left(\mathrm{N} \cdot \mathrm{s} \cdot \mathrm{m}^{-1}\right)\end{array}$ & - & $-2.35 \times 10^{5}$ & $-2.75 \times 10^{5}$ \\
\hline $\begin{array}{l}\text { 阻尼系数 } \\
c_{y y} /\left(\mathrm{N} \cdot \mathrm{s} \cdot \mathrm{m}^{-1}\right)\end{array}$ & - & $9.61 \times 10^{5}$ & $1.21 \times 10^{6}$ \\
\hline
\end{tabular}

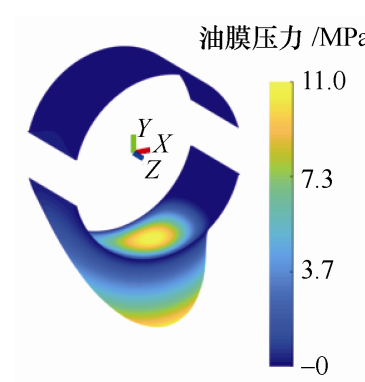

(a) 油膜压力

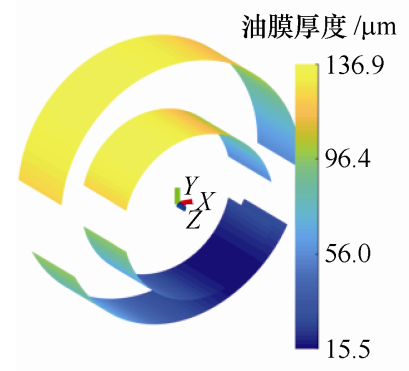

(c) 油膜厚度

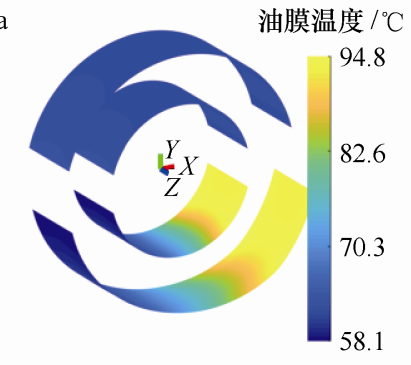

(b) 油膜温度

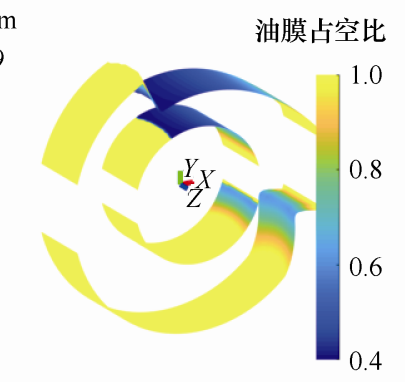

(d) 油膜占空比
图 2 无剪切稀化效应的轴承油膜特性分布

由表 3 可知, 对比无剪切稀化作用的理论和试 验分析结果理论与试验结果可见，二者总体吻合良 好, 偏心率、最小油膜厚度功耗和最高温度误差均 在 $5 \%$ 以内，说明了理论分析方法的正确性。注意到 端泄流量误差较大, 这可能是由于试验过程中由轴 承两侧泄出的润滑油重新进油瓦块上游所造成的。 对比有无剪切稀化效应的理论结果可见: 剪切稀化 
效应导致最小油膜厚度降低, 最高温度升高, 流量 增加, 功耗略有降低, 主刚度和主阻尼略有增加。

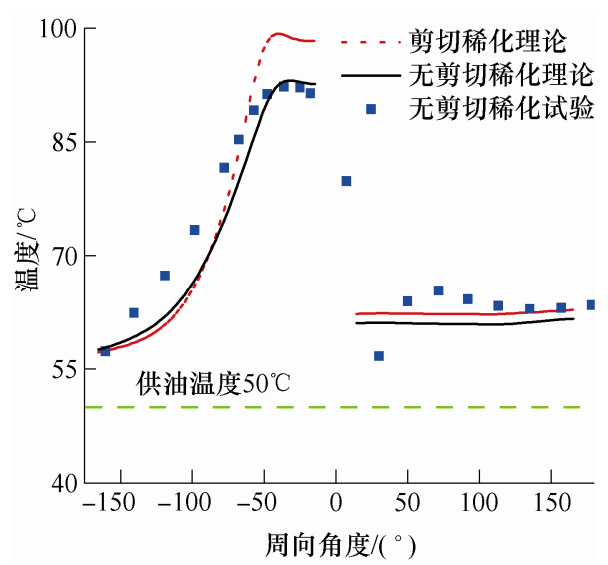

图 3 轴向中心线展开油膜温度

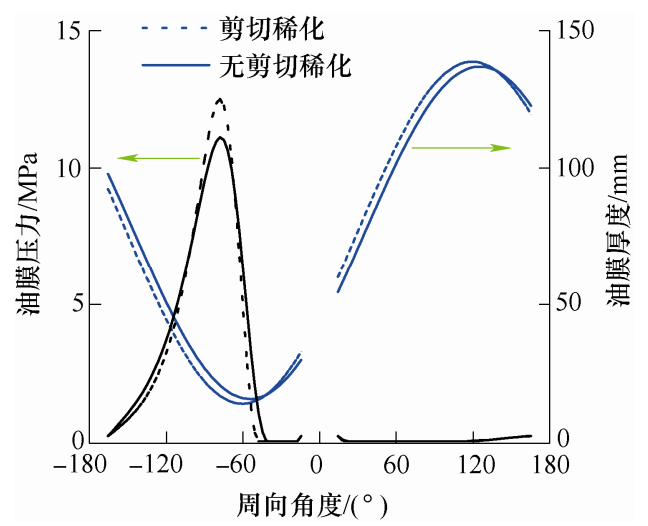

图 4 轴向中心线展开油膜压力与油膜厚度

由图 2 可见, 油膜压力和油膜厚度分布符合预期; 由图 $2 b$ 可见, 油膜温度在周向变化剧烈, 而轴向方向 温度上几乎不变, 同时由于热油的回流现象, 油膜最 低温度明显高于进油温度; 由图 $2 \mathrm{~d}$ 可见, 最低的油膜 占空比出现上瓦 $130^{\circ}$ 处, 最小值为 0.4 。值得注意的 是, 油膜占空比对摩擦力和功耗的计算精度具有重要 影响。由图 3 可见, 温度场的理论计算结果和试验结 果吻合良好, 说明理论模型的正确性; 同时可以发现, 剪切稀化效应导致油膜温升更高。由图 4 可见, 剪切 稀化导致最小油膜厚度减小, 最大油膜压力升高。

由表 2、图 3 与图 4 可知, 在本工况下剪切稀 化虽然可使功耗略有降低, 但在总体上恶化了轴承 其他关键性能参数, 如显著减小油膜厚度、增加油 膜温度、增加流量。原因如下: 本算例属于重载工 况(比压 $3.25 \mathrm{MPa}$, 偏心率大于 0.8 ), 轴承的主要矛 盾是载荷高导致油膜厚度过小和温度过高, 而剪切 稀化效应减小了润滑油黏度, 削弱了轴承承载力, 进一步减小了最小油膜厚度; 另外, 从摩擦生热角 度来看, 黏度下降导致偏心率增加, 油膜压力显著 增加, 压差流造成的生热效应的增加, 即式(6)右端 第 3、4 项增加, 抵消并超过了剪切流生热效应的降
低，进而造成了最高温度升高。

\section{3 转速的影响}

本节探讨进油温度为 $50{ }^{\circ} \mathrm{C}$, 载荷为 $9430 \mathrm{~N}$, 转速为 $1000 \sim 8000 \mathrm{r} / \mathrm{min}$ 时剪切稀化对轴承关键 润滑特性参数的影响规律。图 5 对比了有无剪切稀 化效应时油膜最高温度随转速的变化。图 6 对比了 有无剪切稀化效应时轴承功耗随转速的变化。图 7 对比了有无剪切稀化效应时轴承端泄流量随转速的 变化。图 8 对比了有无剪切稀化效应时最大油膜压 力和最小油膜厚度随转速的变化。

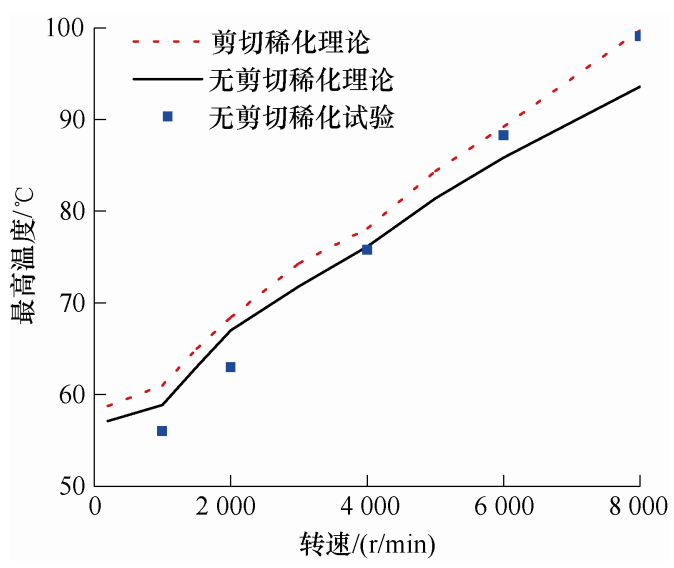

图 5 最高温度随转速的变化

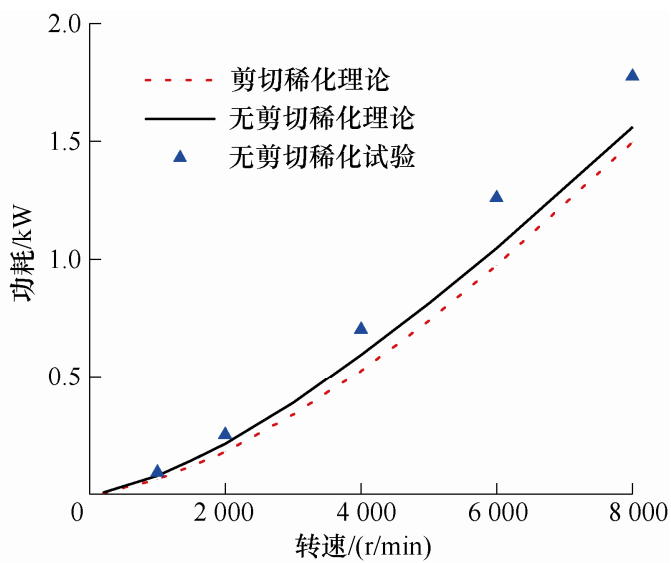

图 6 功耗随转速的变化

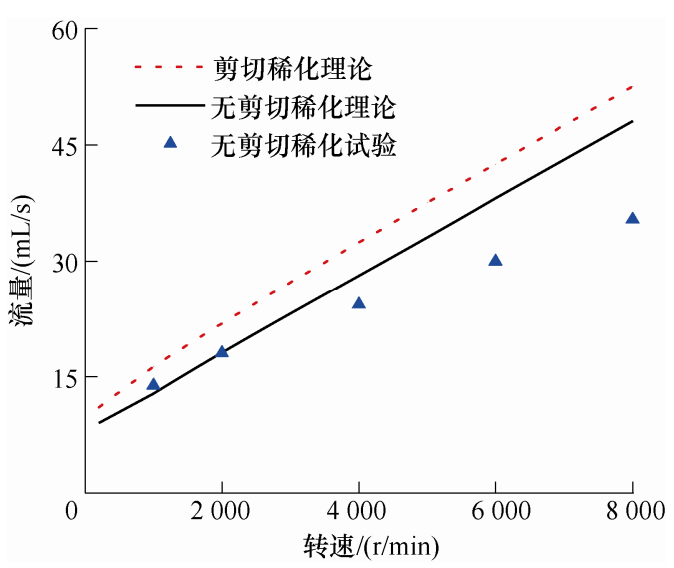

图 7 流量随转速的变化

由图 5 可见, 最高温度的理论与试验结果总体 
吻合良好, 误差在 $5{ }^{\circ} \mathrm{C}$ 以内, 随着转速的增加, 油 膜温度几乎线性增加, 说明转速是影响油膜温度的 重要因素; 同时注意到, 在所有转速范围内, 剪切 稀化都使最高温度增加了 $2 \sim 5{ }^{\circ} \mathrm{C}$ 。

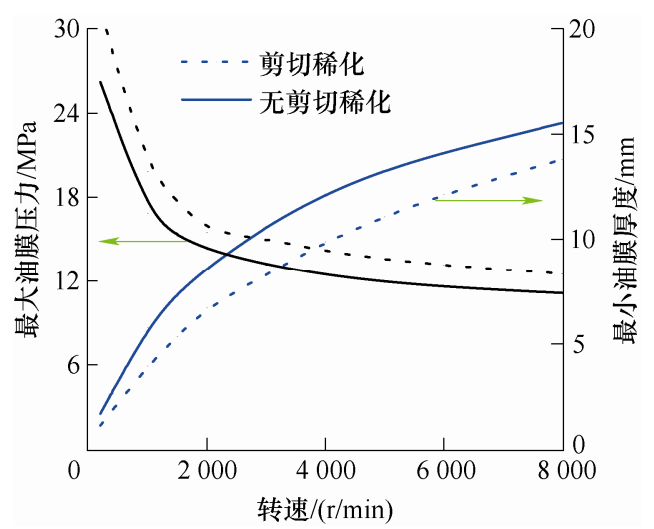

图 8 最小油膜厚度与最大油膜压力随转速的变化

由图 6 可见, 随着转速的增加轴承功耗快速增 加。在低速下, 理论与试验结果吻合很好, 随着转 速的升高, 二者偏差略微增大, 但最大偏差不超过 $10 \%$, 这可能是试验中的轴颈的摚油损耗所导致的。 同时可发现, 在所有转速下剪切稀化均使功耗略有 减低, 但降低幅值不超过 5\%。由图 7 可见, 随着 的升高, 端泄流量快速增加, 低速下端泄流量转速 的理论与试验结果吻合较好, 随着转速的升高, 理 论计算结果逐渐高估了端泄流量。8 $000 \mathrm{r} / \mathrm{min}$ 时理论 高估流量可达 $25 \%$, 这可能是由于实际的轴承两侧 存在端盖密封, 端泄流量由于高速卷吸作用重新进 入瓦块入口所导致的; 同时可发现, 在所有转速下 剪切稀化使流量均略有增加, 平均增加约为 $10 \%$ 。

由图 8 可见, 由于低速剪切率低, 剪切稀化效 应不明显, 最小油膜厚度相差不大, 随着转速的提 高, 剪切稀化效应增强, 最小油膜厚度较常规润滑 油显著降低, 当转速超过 $2000 \mathrm{r} / \mathrm{min}$ 时, 平均降低 幅值约为 $10 \%$ 。同时, 在所有转速下剪切稀化均使 最大油膜压力增加, 平均增加幅值约为 $12 \%$ 。

由图 5 8 可知, 重载工况下, 除略微降低了轴 承功耗外, 剪切稀化效应恶化了轴承的主要静态润 滑性能, 体现在降低最小油膜厚度、增加最大油膜 压力和增加端泄流量。由本例可见, 剪切稀化效应 并不总是对润滑体现正面积极影响。

\section{4 载荷的影响}

本节探讨进油温度为 $50^{\circ} \mathrm{C}$, 转速为 $8000 \mathrm{r} / \mathrm{min}$, 载荷为 $1 \sim 10 \mathrm{kN}$ 时(比压 $0.34 \sim 3.4 \mathrm{MPa}$ ) 剪切稀化 对轴承的主要润滑特性参数的影响规律。图 9 对比 了有无剪切稀化效应时最大油膜压力和最小油膜厚 度随载荷的变化。图 10 对比了有无剪切稀化效应时 最高温度随载荷的变化。图 11 对比了有无剪切稀化
效应时功耗随载荷的变化。

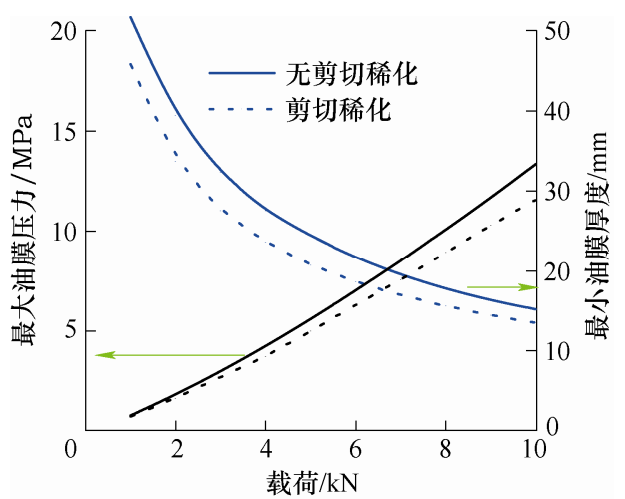

图 9 最小油膜厚度与最大油膜压力随载荷的变化

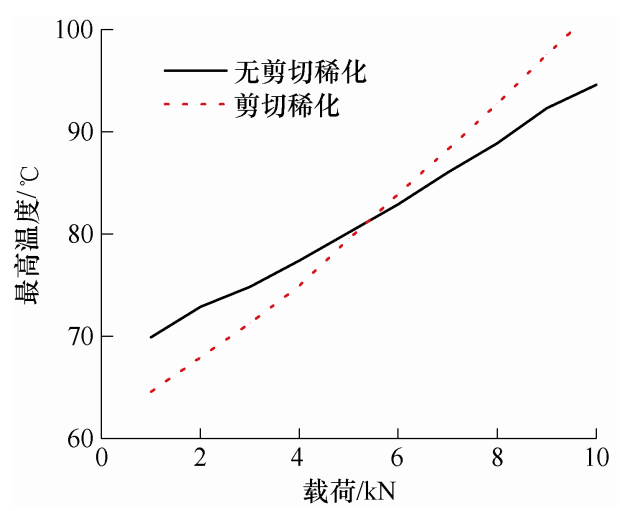

图 10 高温度随载荷的变化

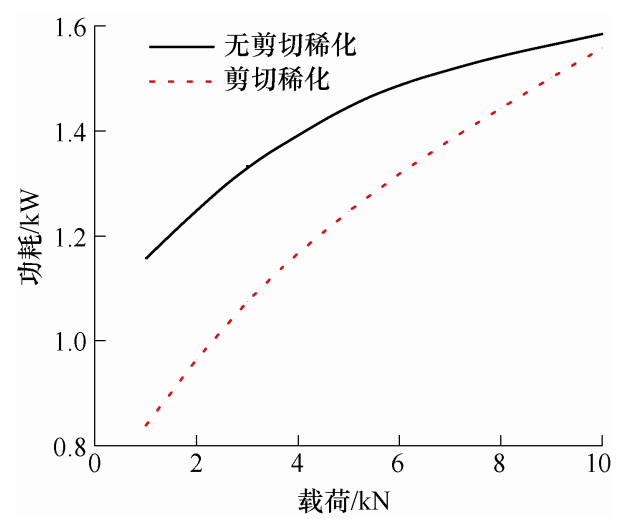

图 11 耗随载荷的变化

由图 9 可见, 首先, 随着载荷的增加, 最大油 膜压力几乎线性增加, 而最小油膜厚度减小幅度较 为缓慢, 说明油膜刚度随载荷的增加而增加。其次, 剪切稀化使最小油膜厚度略有降低。最后, 轻载工 况最大油膜压力差异不大, 随载荷增加, 剪切稀化 效应所造成的最大油膜压力的差异逐渐增加。由图 10 可见, 油膜温度随载荷的增加而增加; 同时注意 到, 轻载工况剪切稀化使油膜的最高温度降低, 而 重载工况情况则相反, 这说明剪切稀化使油膜温度 对载荷的变化更加敏感, 具有剪切稀化效应的润滑 油更适用于轻载工况。由图 11 可见, 功耗随载荷的 增加而增加, 而具有剪切稀化效应的功耗对载荷更 
加敏感。在轻载工况, 剪切稀化效应显著减低了摩 擦功耗, 最高可达 $27.4 \%$, 随着载荷的增加剪切稀 化降低功耗的优势逐渐消失。

由图 9 11 可知, 载荷对剪切稀化效应的强弱 具有重要影响。轻载工况下应用剪切稀化效应对轴 承润滑性能体现为正面作用(如温升和功耗均显著 降低); 而重载工况使用主要体现为负面影响(如油 膜厚度低、温升高、油膜压力高)。原因在于, 轻载 工况油膜压力和压力梯度较小, 压差流影响有限, 决定温升和功耗的主要因素是剪切流, 剪切稀化使 黏度降低, 故温升和功耗均有显著降低; 反之, 重 载工况, 最小油膜厚度薄, 局部剪切率很高, 剪切 稀化效应导致局部黏度明显降低, 进一步削弱了油 膜的承载力, 增加功耗和温升, 降低油膜厚度, 形 成恶性循环。由本节讨论可见, 载荷条件是影响剪 切稀化效应的重要因素, 在轻载工况剪切稀化效应 对轴承具有正面影响, 反之亦然。

\section{5 温度的影响}

本节探讨转速为 $8000 \mathrm{r} / \mathrm{min}$, 载荷为 $9.43 \mathrm{kN}$ 下, 进油温度为 $20 \sim 60{ }^{\circ} \mathrm{C}$ 时, 有无剪切稀化效应 时轴承润滑性能的变化规律。图 12 对比了有无剪切 稀化效应时最大油膜压力和最小油膜厚度随进油温 度的变化。图 13 对比了有无剪切稀化效应时功耗和 流量随进油温度的变化。

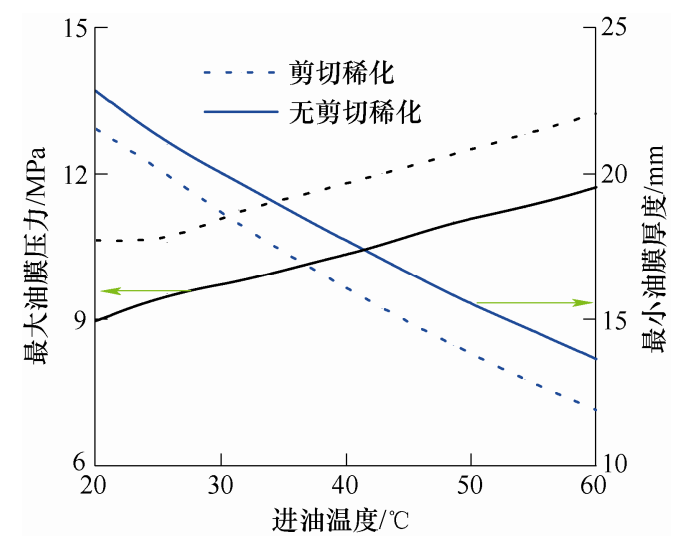

图 12 小油膜厚度与最大油膜压力随进油温度的变化

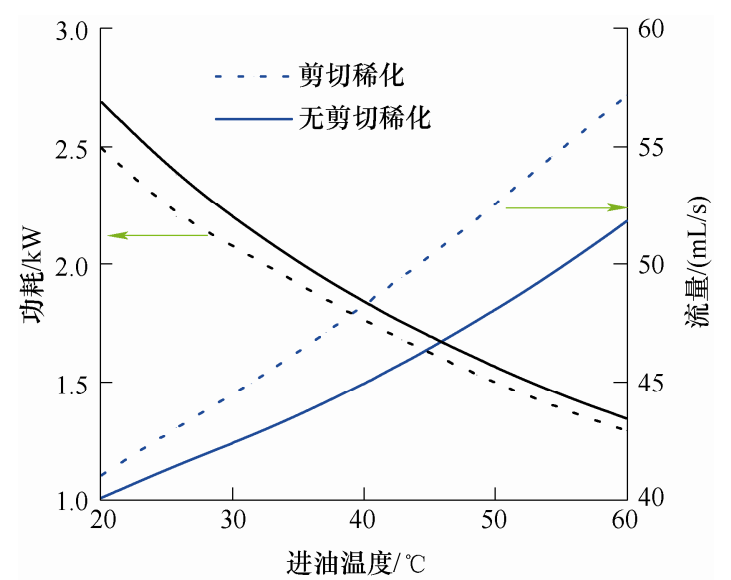

图 13 耗和流量随进油温度的变化
由 12 图可见, 随着进油温度的增加, 最大油膜 压力逐渐增加, 最小油膜厚度逐渐降低。同时, 剪 切稀化使油膜压力平均增加 $12 \%$, 使油膜厚度平均 降低 $10 \%$, 但不同进油温度下, 有无剪切稀化效应 下的油膜压力和油膜厚度差异不大, 说明剪切稀化 效应对进油温度不敏感。由图 13 可见，随着进油温 度的升高, 功耗显著降低, 流量显著增加。同时注 功耗和流量而言, 增加进油温度 $5^{\circ} \mathrm{C}$ 即可达到与包 含剪切稀化效应相似的作用效果。由第 3.5 节讨论 可知, 剪切稀化效应对进油温度不敏感, 提高进油 温度可达到与剪切稀化效应类似的效果。

\section{6 热分析模型的探讨}

大量理论计算说明剪切稀化效应可以显著降低

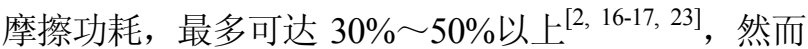
目前已有的试验数据 ${ }^{[5,19-20,22,24]}$ 并没有证明剪切稀化 确具有如此显著的降低功耗的作用, 我们尝试从理论 分析方法角度产生上述矛盾的原因。本节采用等温模 型和绝热模型分析剪切稀化对轴承性能的影响, 设定 载荷为 $9430 \mathrm{~N}$, 其中等温模型设定等效工作温度为 $70{ }^{\circ} \mathrm{C}$, 绝热模型进油温度为 $50^{\circ} \mathrm{C}$ 。图 14 对比了基于 等温和绝热模型的有无剪切稀化效应时最小油膜厚 度随转速的变化。图 15 对比了基于等温和绝热模型 的有无剪切稀化效应时功耗随转速的变化。

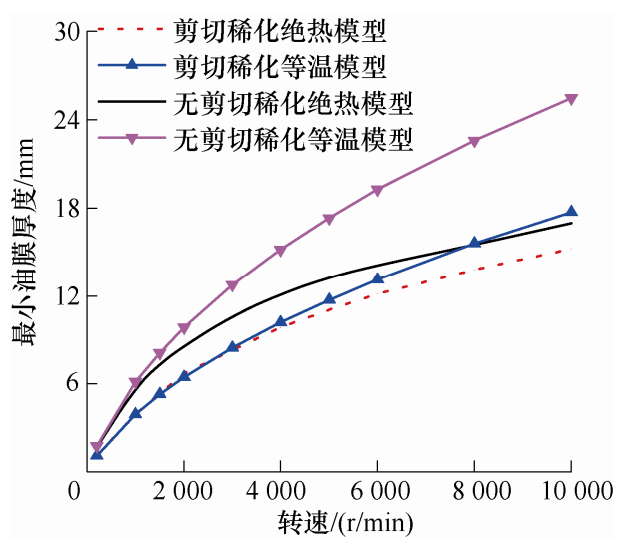

图 14 分析模型对最小油膜厚度的影响

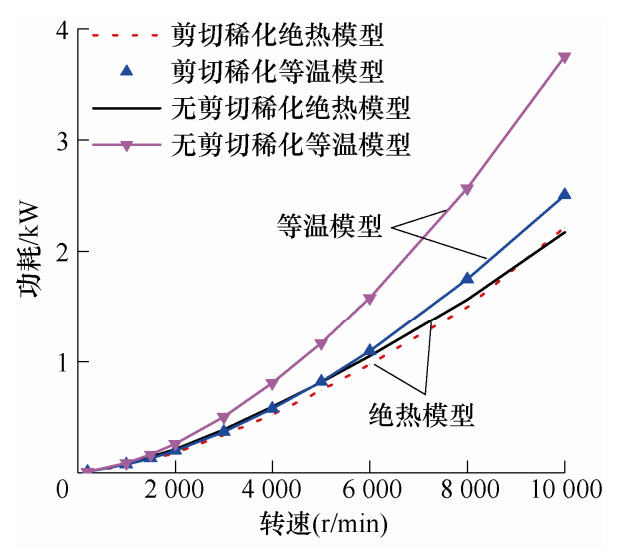

图 15 热分析模型对功耗的影响 
由图 14 可见, 随着转速的升高, 基于等温模型, 有无剪切稀化效应最小油膜厚度的差异逐步扩大, $8000 \mathrm{r} / \mathrm{min}$ 时剪切稀化使油膜厚度降低 30.6\%, 而基 于绝热模型, 有无剪切稀化效应油膜厚度的差异随转 速变化不大, 平均降低仅约 10\%。由图 15 可见, 基 于等温模型, 剪切稀化效应最多可使摩擦功耗降低 $30 \%$, 而基于绝热模型, 剪切稀化效应对功耗的影响 小于 $5 \%$ 。需要注意, 本节采用的是重载工况, 根据 第 3.4 节分析结果可知, 在轻载工况下剪切稀化对功 耗的降低作用将有所增加, 但总体而言, 等温模型均 高估了剪切稀化的作用, 因此对于使用多级润滑油的 滑动轴承的设计与计算均应选用绝热模型。

综上, 对于剪切稀化效应对轴承功耗的理论和 试验结果存在较大偏差的问题, 我们认为原因有两 点: (1) 已有理论分析往往采用给定偏心 ${ }^{[1-4,6,9,14-15]}$ 而非给定载荷, 显然偏心相同并不意味着载荷工况 相同, 这种分析方法忽视了剪切稀化效应对润滑性 能影响强弱与工况强烈相关的特点, 造成了非同工 况性能对比; (2) 温度计算模型对剪切稀化效应具有 至关重要的影响, 传统的等温模型 ${ }^{[16,19-20,23]}$ 严重高 估了剪切稀化效应的影响。

\section{4 结论}

(1) 剪切稀化效应是剪切率和温度的强函数, 剪 切稀化效应对润滑性能影响强弱与工况强烈相关。

(2) 转速是影响剪切稀化效应的重要因素, 高 速下对轴承性能影响显著, 但对轴承的性能的影响 规律与其他因素强耦合。

（3）载荷是影响剪切稀化效应的重要因素, 轻 载工况剪切稀化效应对轴承润滑性能更多展示出正 面作用, 重载工况更多展现负面作用, 故具有剪切 稀化效应的润滑油更适用于轻载工况。

(4) 剪切稀化效应对进油温度不敏感, 提高进 油温度可达到与剪切稀化效应相似的效果。

(5) 定偏心计算和等温模型严重高估了剪切稀 化效应对轴承润滑性能的影响, 导致轴承功耗的理 论和试验存在较大偏差。

\section{参 考 文 献}

[1] ZHANG C, CHENG H S. Transient non-Newtonian thermohydrodynamic mixed lubrication of dynamically loaded journal bearings[J]. Journal of Tribology, 2000, 122(1): 156-161.

[2] GECIM B A. Non-Newtonian effects of multigrade oils on journal bearing performance[J]. Tribology Transactions,
1990, 33(3): 384-394.

[3] TANNER R I. A short-bearing solution for pressure distribution in a non-Newtonian lubricant[J]. Journal of Applied Mechanics, 1964, 31(2): 350-351.

[4] WADA S, HAYASHI H. Hydrodynamic lubrication of journal bearings by Pseudo-Plastic lubricants : Part 1, Theoretical studies[J]. Bulletin of JSME, 1971，14(69): 268-278.

[5] WADA S, HAYASHI H. Hydrodynamic lubrication of journal bearings by Pseudo-Plastic lubricants : Part 2, Experimental studies[J]. Bulletin of JSME, 1971, 14(69): 279-286.

[6] SWAMY S T N, PRABHU B S, RAO B V A. Calculated load capacity of non-Newtonian lubricants in finite width journal bearings[J]. Wear, 1975, 31(2): 277-285.

[7] SWAMY S T N, PRABHU B S, RAO B V A. Stiffness and damping characteristics of finite width journal bearings with a non-Newtonian film and their application to instability prediction[J]. Wear, 1975, 32(3): 379-390.

[8] SWAMY S T N, PRABHU B S, RAO B V A. Steady state and stability characteristics of a hydrodynamic journal bearing with a non-Newtonian lubricant[J]. Wear, 1977, 42(2): 229-244.

[9] DIEN I K, ELROD H G. A generalized steady-state Reynolds equation for non-Newtonian fluids, with application to journal bearings[J]. Journal of Lubrication Technology, 1983, 105(3): 385-390.

[10] JIIN-YUH J, CHONG-CHING C. Adiabatic analysis of finite width journal bearings with non-Newtonian lubricants[J]. Wear, 1988, 122(1): 63-75.

[11] SHEAUMING J U, WENG C. Thermohydrodynamic analysis of finite-width journal bearings with non-Newtonian lubricants[J]. Wear, 1994, 171(s 1-2): 41-49.

[12] CROSS M M. Rheology of non-Newtonian fluids: A new flow equation for pseudoplastic systems[J]. Journal of Colloid Science, 1965, 20(5): 417-437.

[13] PARANJPE R S. Analysis of non-Newtonian effects in dynamically loaded finite journal bearings including mass conserving cavitation[J]. Journal of Tribology, 1992, 114(4): 736-744.

[14] WANG $\mathrm{P}$, KEITH $\mathrm{T} \mathrm{G}$, VAIDYANATHAN $\mathrm{K}$. Non-Newtonian effects on the performance of dynamically loaded elliptical journal bearings using a mass-conserving finite element cavitation algorithm[J]. Tribology Transactions, 2001, 44(4): 533-542.

[15] JANG J Y, KHONSARI M. Thermohydrodynamic analysis of journal bearings lubricated with multigrade oils[J]. Computer Modeling in Engineering and Sciences, 2002, 3(4): 455-464. 
[16] FATU A, HAJJAM M, BONNEAU D. Analysis of non-Newtonian and piezoviscous effects in dynamically loaded connecting-rod bearings[J]. Proceedings of the Institution of Mechanical Engineers, Part J: Journal of Engineering Tribology, 2005, 219(3): 209-224.

[17] BUKOVNIK S, OFFNER G, ČAIKA V, et al. Thermo-elasto-hydrodynamic lubrication model for journal bearing including shear rate-dependent viscosity[J]. Lubrication Science, 2007, 19(4): 231-245.

[18] 张振山. 计入非牛顿、变形及表面形貌效应的动载轴承 热流体动力润滑分析[D]. 上海: 上海交通大学, 2014 . ZHANG Zhenshan. Analysis of thermo hydrodynamic performance of dynamically loaded bearings in consideration of non-Netonian, deformation and surface topograhpy[D]. Shanghai: Shanghai Jiao Tong University, 2014.

[19] SANDER D E, ALLMAIER H, PRIEBSCH H H, et al. Impact of high pressure and shear thinning on journal bearing friction[J]. Tribology International, 2015, 81(1): 29-37.

[20] 宁李谱, 宁欣, 孟祥慧. 考虑润滑油剪切稀化效应的活 塞裙部混合润滑性能 $[\mathrm{J}]$. 上海交通大学学报, 2018 , 52(5): 511-517.

NING Lipu, NING Xin, MENG Xianghui. Piston skirt mixed lubrication analysis considering the effects of lubricant shear-thinning[J]. Journal of Shanghai Jiao Tong University, 2018, 52(5): 511-517.

[21] 汤占岐. 关节轴承非牛顿流体润滑与摩擦研究及寿命 预测[D]. 合肥：合肥工业大学, 2017.

TANG Zhanqi. Research on lubrication and friction of spherical plain bearing lubricated with non-Newtonian fluid and service life prediction[D]. Hefei : Hefei University of Technology, 2017.

[22] MARX N, FERNÁNDEZ L, BARCELÓ F, et al. Shear thinning and hydrodynamic friction of viscosity modifier-containing oils. Part I : Shear thinning behaviour[J]. Tribology Letters, 2018, 66(3): 92-93.

[23] MARX N, FERNÁNDEZ L, BARCELÓ F, et al. Shear thinning and hydrodynamic friction of viscosity modifier-containing oils. part II: impact of shear thinning on journal bearing friction[J]. Tribology Letters, 2018, 66(3): 91-92.

[24] VLADESCU S, MARX N, FERNÁNDEZ L, et al. Hydrodynamic friction of viscosity-modified oils in a journal bearing machine[J]. Tribology Letters, 2018, 66(4): $127-128$

[25] BOOSER E R. Theory and practice of tribology: Volume II: Theory and design[M]. Boca Raton, Fla.: CRC Press, 1984.

[26] KUMAR A, BOOKER J F. A finite-element cavitation algorithm[J]. Journal of Tribology-Transactions of the ASME, 1991, 113(2): 276-286.

[27] FITZGERALD M K, NEAL P B. Temperature distributions and heat transfer in journal bearings[J]. Journal of Tribology, 1992, 114(1): 122-130.

作者简介: 裴世源(通信作者), 男, 1983 年出生, 博士, 副研究员。主 要研究方向为滑动轴承与转子动力学。

E-mail: peishiyuan@xjtu.edu.cn

洪军, 男, 1968 年出生, 博士, 教授, 博士研究生导师。主要研究方向 为精准数字装配理论与技术、高性能数控机床数字化设计与制造技术。 E-mail: hongjun@xjtu.edu.cn 